\title{
Proceedings for the 14th European Powder Diffraction Conference published online as a 2015 Special Supplement Issue of Powder Diffraction Journal
}

European Powder Diffraction Conference (EPDIC 14) was held in Aarhus, Denmark on 15-18 June 2014. A beautiful city, pleasant weather, and an outstanding conference all made for a wonderful experience. As part of EPDIC 14, the International Centre for Diffraction Data (ICDD) sponsored the publication of the conference proceedings. Through Cambridge University Press, the EPDIC 14 proceedings with 21 papers (see Table I) are being published online (http://jour nals.cambridge.org/action/displayJournal?jid=PDJ).

EPDIC 14 attendees receive free access to the proceedings for 2 years and subscribers to Powder Diffraction also have access. Anyone without a PDJ subscription or did not attend
EPDIC 14 can purchase any of the articles for a fee. I would like to thank the EPDIC 14 Proceedings Editors Jens-Erik Jorgensen, Paolo Scardi, and Michela Brunelli for their efforts in getting all of the papers together, reviewed, and approved for publication. A special thanks to Nicole Ernst-Boris, Powder Diffraction Managing Editor, for her diligence in coordinating the submission of manuscripts to Cambridge University Press (Photo 1).

Tom Blanton

International Centre for Diffraction Data

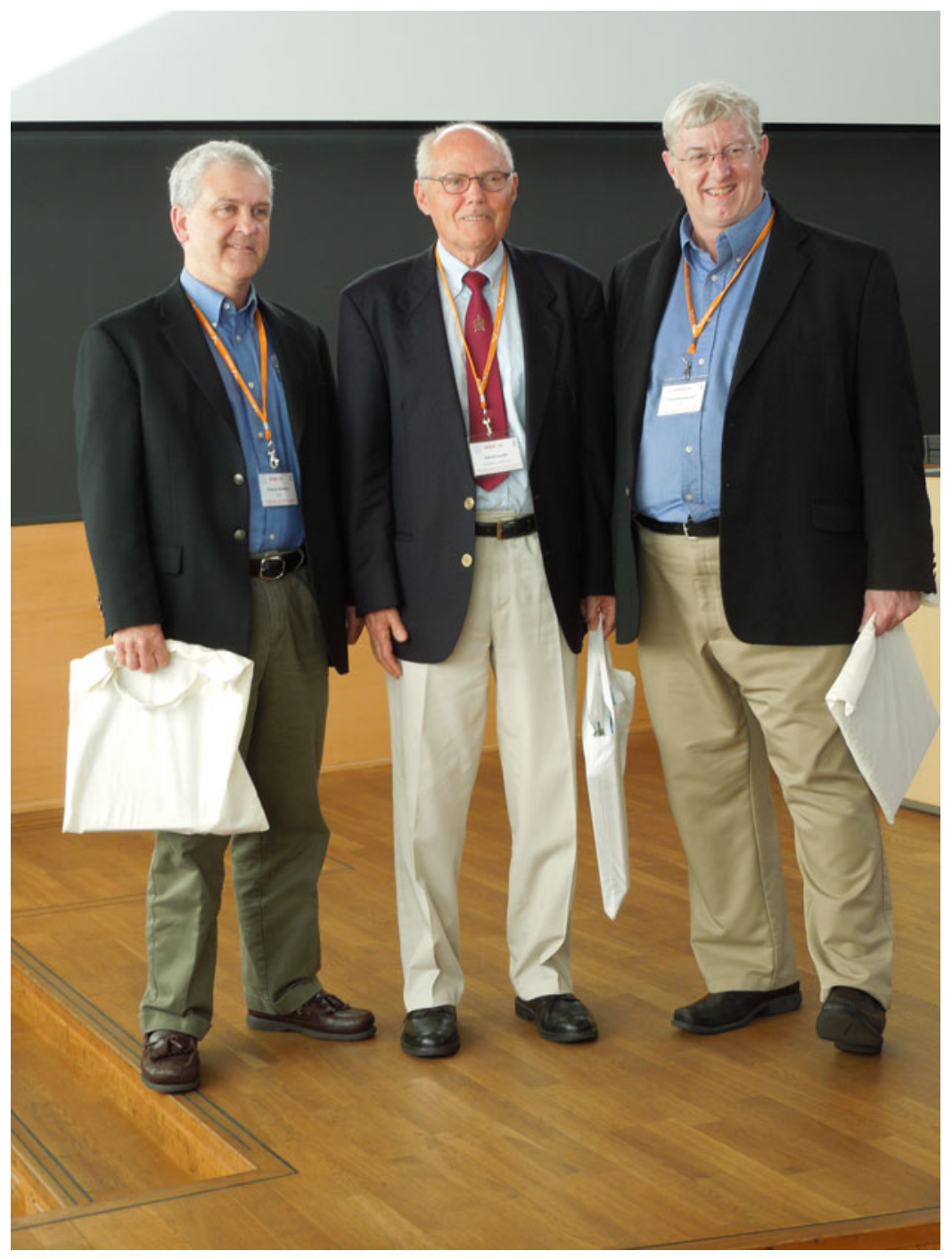

Photo 1. (Color online) A highlight of the EPDIC 14 meeting was the presentation by the EPDIC Committee, the EPDIC Award for Distinguished Powder Diffractionists to Professor Daniel Louer (Rennes, France). Professor Louer is also an ICDD Distinguished Fellow. Shown here are Daniel (middle) being congratulated by ICDD Principal Scientist Tom Blanton (left) and ICDD Executive Director Tim Fawcett (right). 
TABLE I. Published papers in EPDIC 14 proceedings.

\begin{tabular}{|c|c|c|c|}
\hline Manuscript title & Authors & Manuscript title & Authors \\
\hline $\begin{array}{l}\text { Total scattering experiments on glass } \\
\text { and crystalline materials at the } \\
\text { ESRF on the ID11 Beamline }\end{array}$ & $\begin{array}{l}\text { Bernasconi, Andrea; Wright, } \\
\text { Jonathan; Harker, Nicholas }\end{array}$ & $\begin{array}{l}\text { A comparison of total scattering data } \\
\text { from various sources: the case of a } \\
\text { nanometric spinel }\end{array}$ & $\begin{array}{l}\text { Confalonieri, Giorgia; Dapiaggi, } \\
\text { Monica; Sommariva, Marco; } \\
\text { Gateshki, Milen; Fitch, Andy; } \\
\text { Bernasconi, Andrea }\end{array}$ \\
\hline $\begin{array}{l}\text { Mechanism and kinetics of early } \\
\text { transition metal hydrides, oxides } \\
\text { and chlorides to enhance hydrogen } \\
\text { release and uptake properties of } \\
\mathrm{MgH}_{2}\end{array}$ & $\begin{array}{l}\text { Korablov, Dmytro; Nielsen, } \\
\text { Thomas; Besenbacher, } \\
\text { Flemming; Jensen, Torben }\end{array}$ & $\begin{array}{l}\text { The water retention mechanism of a } \mathrm{Cs}^{+} \\
\text {and } \mathrm{Na}^{+} \text {exchanged montmorillonite: } \\
\text { effect of relative humidity and ionic } \\
\text { radius }\end{array}$ & $\begin{array}{l}\text { Ammar, Marwa; Oueslati, Walid; } \\
\text { Chorfi, Nejmeddine; Amara, } \\
\text { Abdesslem }\end{array}$ \\
\hline $\begin{array}{l}\text { Thin film morphologies of block } \\
\text { copolymers with nanoparticles }\end{array}$ & $\begin{array}{l}\text { Jehnichen, Dieter; Pospiech, Doris; } \\
\text { Friedel, Peter; He, Guping; Sepe, } \\
\text { Alessandro; Zhang, Jianqi; } \\
\text { Papadakis, Christine; Taurino, } \\
\text { Rosa; Perlich, Jan }\end{array}$ & $\begin{array}{l}\text { Stress-induced phase transformations in } \\
\text { thermally cycled superelastic NiTi } \\
\text { alloys: in situ X-ray diffraction studies. }\end{array}$ & $\begin{array}{l}\text { Polatidis, Efthymios; Zotov, } \\
\text { Nikolay; Mittemeijer, Eric }\end{array}$ \\
\hline $\begin{array}{l}\text { Trace phase detection and strain } \\
\text { characterization from serial XFEL } \\
\text { crystallography of a } \operatorname{Pr}_{0.5} \mathrm{Ca}_{0.5} \mathrm{MnO}_{3} \\
\text { Powder }\end{array}$ & $\begin{array}{l}\text { Beyerlein, Kenneth; Jooss, } \\
\text { Christian; Barty, Anton; Bean, } \\
\text { Richard; Boutet, Sébastien; } \\
\text { Dhesi, Sarnjeet; Doak, R. Bruce; } \\
\text { Först, Michael; Galli, Lorenzo; } \\
\text { Kirian, Richard; Kozak, Joseph; } \\
\text { Lang, Michael }\end{array}$ & $\begin{array}{l}\text { Study of residual stresses, microstructure } \\
\text { and hardness in } \mathrm{FeB} \text { and } \mathrm{Fe}_{2} \mathrm{~B} \\
\text { ultra-hard layers }\end{array}$ & $\begin{array}{l}\text { Pala, Zdenek; Fojtikova, Jaroslava; } \\
\text { Koubsky, Tomas; Musalek, } \\
\text { Radek; Strasky, Josef; Capek, } \\
\text { Jiri; Kyncl, Jiri; Beranek, Libor; } \\
\text { Kolarik, Kamil }\end{array}$ \\
\hline $\begin{array}{l}\text { Nanocrystalline and } \\
\text { stacking-disordered } \beta \text {-cristobalite } \\
\mathrm{AlPO}_{4}-\text { the now deciphered main } \\
\text { constituent of a municipal sewage } \\
\text { sludge ash from a full-scale } \\
\text { incineration facility }\end{array}$ & $\begin{array}{l}\text { Peplinski, Burkhard; Adam, } \\
\text { Christian; Adamczyk, Burkart; } \\
\text { Müller, Ralf; Michaelis, Matthias; } \\
\text { Krahl, Thoralf; Emmerling, } \\
\text { Franziska }\end{array}$ & $\begin{array}{l}\text { Corrugations of the basal planes in } \\
\text { hexagonal boron nitride and their } \\
\text { impact on the phase transition to cubic } \\
\text { boron nitride }\end{array}$ & $\begin{array}{l}\text { Schimpf, Christian; Schwarz, } \\
\text { Marcus; Lathe, Christian; } \\
\text { Kroke, Edwin; Rafaja, David }\end{array}$ \\
\hline $\begin{array}{l}\text { Using synergy of experimental and } \\
\text { computational techniques to solve } \\
\text { monomer-trimer dilemma }\end{array}$ & $\begin{array}{l}\text { Sisak Jung, Dubravka; Jović, Ozren; } \\
\text { Kalinovičić, Petra; Primožič, } \\
\text { Ines; Hrenar, Tomica }\end{array}$ & $\begin{array}{l}\text { Ferromagnetic ordering in } \mathrm{La}_{0.7} \mathrm{Sr}_{0.3} \mathrm{Mn}_{3} \\
\quad+0.85 \mathrm{Nb}_{5}+0.15 \mathrm{O}_{3} \text { manganite }\end{array}$ & $\begin{array}{l}\text { Troyanchuk, Igor; Bushinsky, } \\
\text { Maxim; Efimov, Vadim; Schorr, } \\
\text { Susan; Ritter, Clemens; } \\
\text { Sikolenko, Vadim }\end{array}$ \\
\hline $\begin{array}{l}\text { New type of versatile neutron } \\
\text { diffractometer with a double-crystal } \\
\text { (DC) monochromator system }\end{array}$ & Mikula, Pavol & $\begin{array}{l}\text { Crystal structure of new phosphates } \\
\mathrm{Ca}_{9 x} \mathrm{~Pb}_{x} \mathrm{Eu}\left(\mathrm{PO}_{4}\right)_{7} \text { from Rietveld } \\
\text { refinement }\end{array}$ & $\begin{array}{l}\text { Deyneko, Dina; Stefanovich, } \\
\text { Sergey; Lazoryak, Bogdan }\end{array}$ \\
\hline $\begin{array}{l}\text { Toward a reference material for line } \\
\text { profile analysis }\end{array}$ & $\begin{array}{l}\text { Troian, Andrea; Rebuffi, Luca; } \\
\text { Leoni, Matteo; Scardi, Paolo }\end{array}$ & $\begin{array}{l}\text { Temperature dependence of the inversion } \\
\text { degree in three-cation spinel solid } \\
\text { solutions experimental evaluation } \\
\text { by XRD }\end{array}$ & Podwórny, Jacek \\
\hline $\begin{array}{l}\text { Analysis of preferential orientation in } \\
\text { zirconium samples deformed by } \\
\text { uniaxial tension using neutron and } \\
\text { X-ray diffraction }\end{array}$ & Kucerakova, Monika & $\begin{array}{l}\text { Quantification of stacking disordered } \\
\text { Si-Al layer silicates by the Rietveld } \\
\text { method: application to exploration for } \\
\text { high-sulphidation epithermal gold } \\
\text { deposits }\end{array}$ & $\begin{array}{l}\text { Ufer, Kristian; Kleeberg, } \\
\text { Reinhard; Monecke, Thomas }\end{array}$ \\
\hline \multirow[t]{2}{*}{$\begin{array}{l}\text { Design and management of a powder } \\
\text { diffraction beamline for Line Profile } \\
\text { Analysis: a realistic ray-tracing } \\
\text { approach }\end{array}$} & $\begin{array}{l}\text { Rebuffi, Luca; Scardi, Paolo; } \\
\text { Sanchez del Rio, Manuel }\end{array}$ & $\begin{array}{l}\text { Size and spatial correlation of defective } \\
\text { domains in Yttrium-Doped } \mathrm{CeO}_{2}\end{array}$ & $\begin{array}{l}\text { Checchia, Stefano; Scavini, } \\
\text { Marco; Allieta, Mattia; Brunelli, } \\
\text { Michela; Ferrero, Claudio; } \\
\text { Coduri, Mauro }\end{array}$ \\
\hline & & $\begin{array}{l}\text { Characterization of hydrochlorothiazide in } \\
\text { solid formulations }\end{array}$ & $\begin{array}{l}\text { Salvi, Simone; Tita, Diego; } \\
\text { Paiva-Santos, Carlos; Antonio, } \\
\text { Selma }\end{array}$ \\
\hline
\end{tabular}

